\title{
A Contribution to Defining the Term 'Definition'
}

\author{
Sead Spuzic and Fons Nouwens \\ Central Queensland University, Rockhampton, Australia
}

s.spuzic@cqu.edu.au f.nouwens@cqu.edu.au

\begin{abstract}
Knowledge is a model that enables premeditated change. Knowledge can be subdivided: the simplest element is information. Information aggregates/relations constitute a concept. Clusters of concepts make theories. Concepts and theories can be further branched into: conclusions, explanations, cognitions, etc, which together belong to a general class of Definition. Definitions are key elements of knowledge. Yet it appears that we do not sufficiently explain the nature of this concept of definition itself.

There is little doubt about the importance of words and languages, however these semantic structures contain some intrinsic ambiguities. Knowledge records grow faster than its substance, and it is increasingly difficult to manipulate and communicate this voluminous structure. The interpretations of terms and knowledge can vary significantly, especially in the multidisciplinary context. Misiformation is exacerbated by vague definitions including synonyms, homonyms and acronyms.
\end{abstract}

This paper attempts to contribute to defining the term 'definition' to alleviate the mentioned problems.

Keywords: definition, knowledge, information, homonymy, synonymy, misinforming

\section{Introduction}

Over time, a massive stock of information has been accumulated; the body of knowledge records is growing faster than the substance of knowledge itself, and it is increasingly difficult to search, manipulate, communicate, and transfer this voluminous structure. It can be questioned whether we are capable of accessing efficiently the whole body of knowledge accumulated by our ancestors.

Academic efforts to categorize sub-domains and fragments of knowledge into specialist communities of practice or disciplines, each with their special language to describe new phenomena, have certainly brought in the tides of progress in understanding our ambient and ourselves. However, the vivisection of our environment, the enclosure within formal domains, has also raised undesired barriers.

Recent trends in science favor the de-specialization of knowledge and collaborative communication between initially separated disci-

Material published as part of this journal, either on-line or in print, is copyrighted by Informing Science. Permission to make digital or paper copy of part or all of these works for personal or classroom use is granted without fee provided that the copies are not made or distributed for profit or commercial advantage AND that copies 1) bear this notice in full and 2) give the full citation on the first page. It is permissible to abstract these works so long as credit is given. To copy in all other cases or to republish or to post on a server or to redistribute to lists requires specific permission from the publisher at Publisher@InformingScience.org plines. However, both trends have uncovered a variety of hindrances to the transfer of knowledge, such as existence of special terms and acronyms used within disciplines previously isolated from each other, as well 
as the appearance of homonyms and synonyms.

The growth of knowledge stock and the occurrence of cross-disciplinary communication are necessary, but not sufficient conditions for further improving knowledge transfer (Cartelli, 2003; Spuzic, 1999; Spuzic \& O’Brien, 2002a).

There is little doubt about the importance of language in human thinking, and its key role in functioning of our civilisation (e.g. in communication and specifically in education). However, it is argued that contemporary languages are imperfect or not sufficiently developed since the explicitness and interpretations of terms and statements can vary significantly. Everyday language has developed from small community languages and dialects originating dynamically. The progress of civilizations coincides with developments of writing and printing. Yet the "dominant" languages we use today contain vestiges of orality and early literacy (Ong, 1982). Indeed Bolter (2001) argues that new developments in the use of information and communication technologies are slow to capitalize on the capabilities these technologies offer because one of the ways cultures adopt these new technologies is by making them imitate earlier technologies like print, writing and even oral techniques. As a result inconsistencies are many.

Ambiguities due to accumulation of synonyms, homonyms and acronyms have become apparent during the development of programs for applications of artificial intelligence. Since the synonyms, homonyms and acronyms bring vagueness and misunderstanding in the communication and interpretation of knowledge, they should be considered as important cases of misinforming.

A further problem is the multiplication of the records of already existing knowledge due to translation into other languages. These translations obviously increase further the amount of formal records. Do we really need to have the same knowledge written in Mandarin, French, Russian, Spanish, and Czech...?

There is no doubting the enormous advantages of the existence of variety of languages - the question is how this treasure can be made more useful.

Knowledge is a model (replica) of some relations that enables realisation of premeditated changes in some relations. A specific piece of knowledge has a context; this framework relates to when and where it can be reliably applied. We can state that we possess certain knowledge only when it actually has enabled repeated performance, irrespective of factors that are not included in formulation of that knowledge.

The body of knowledge can be broken into its structural components in the following manner: the simplest element of knowledge is information. Aggregates made of two or more of these elemental information units, together with their relations, constitute a concept. Clusters of concepts make theories. Information, concept, and theory can be further branched into sub-categories such as: conclusions, explanations, cognitions, contemplations, and all these notions together belong to a more general category of Definition. Definitions represent the bricks in the overall structure of knowledge. Yet it appears that the existing definitions of this very term "DEFINITION" do not sufficiently explain the nature of this crucial concept. Therefore an attempt is made herewith to address phenomena such as homonymy, synonymy and vague definitions to contribute to better defining of the term "DEFINITION".

The physiological basis of human brain functions is still far from being satisfactorily understood. However it is becoming clear that during its evolution, the brain developed a multitude of subsystems to deal with particular tasks (Driscoll, 2000, p.284). There is a certain amount of independence (and interdependence) between these sub-systems. Thus there are major differences between visual and verbal memory and thought processes. Visual processes are older, more primitive and more holistic. We understand 'things' by generalizing relationships, by creating a hierarchy of information, by filtering and selecting significant factors out of the large number of 
possible interactions, by virtue of comparisons and by visualizing eloquent analogies. However, the mode of recording and presenting this knowledge (once it has emerged) present a further crucial factor in communicating this treasure. Verbal definitions only, however logical, are not always sufficient for transmitting knowledge; the means (instruments) for recording and communicating knowledge must to be addressed as well. An old wisdom, that points at the advantages of using the figures and illustrations over the plain words (text and ciphers), carries a sophisticated message about the nature of knowledge itself ad the way human brains work.

\section{Analysis}

\section{Preamble}

Vagueness (ambiguity) and even exaggerated, voluminous presentation, evidently decrease our ability to understand something, hence they can be classified as special cases of misinforming

Understanding our ambient is one of the principal requirements for human survival. In simple terms, if we understand something, we are able then to improve our control over the relevant outcomes; we can predict, affect or induce events, or avoid unfavourable outcomes.

The very fact that we experience a premeditated outcome does not grant that we did actually understand the relevant phenomena - the outcome may be just a consequence of fortunate coincidence, or it may be deliberately produced by an external influence, by means which remain unknown to us.

There is an infinite spectrum of causes that may enhance an ambiguity. For the purpose of this analysis we shall ignore the deliberate vagueness which is produced on purpose.

Our knowledge is often built upon understanding some similar, simpler or contrasting phenomena. Nebulous knowledge of these previous cases often hinders our understanding of the forthcoming phenomena.

The study of meaning, both in general terms and in reference to a specific language, is known as semantics. It embraces the meaningful functions of phonological features, such as intonation, and of grammatical structures and the meanings of individual words. The questions arising from the relations between grammar and meaning are the subjects of continuing controversy today (Cartelli, 2003; "Language," 2003).

\section{Some Aspects of Written Language}

Sharing of knowledge is greatly facilitated by language, though it is possible to share knowledge by demonstration and observation. In oral cultures, the need to memorise information placed a high cognitive overhead on the culture, which tended to be intuitive, community focused and argumentative (Ong, 1982). Writing freed the mind and the culture from the burden of memory, and led to development of more rational, private and reflective thought. Kahneman and Fredrick (2001, p.2) distinguish intuitive (oral) and reflective (written) cognitive processes as follows:

$\begin{array}{cc}\text { Intuitive (oral culture) } & \text { Reflective (written culture) } \\ \text { Automatic } & \text { Controlled } \\ \text { Effortless } & \text { Effortful } \\ \text { Associative } & \text { Deductive } \\ \text { Rapid, parallel } & \text { Slow, serial } \\ \text { Process opaque } & \text { Self-aware } \\ \text { Skilled action } & \text { Rule application }\end{array}$


The transition from orality to literacy the cultural tools required to build technical knowledge, to begin to systematically build knowledge structures. Historically writing is subsequent to speech and presupposes it. Aristotle expressed the relation thus: Speech is the representation of the mental experiences, and writing is the representation of speech.

Alphabetic writing, in which, broadly, consonant and vowel sounds are indicated by letters in sequence, is the most widespread system in use, but it is not the only system, nor is it the earliest one.

Writing appears to have evolved from an extension of pictures: signs that directly and iconically represented some thing or action and then the word that bore that meaning (see Figure 1).
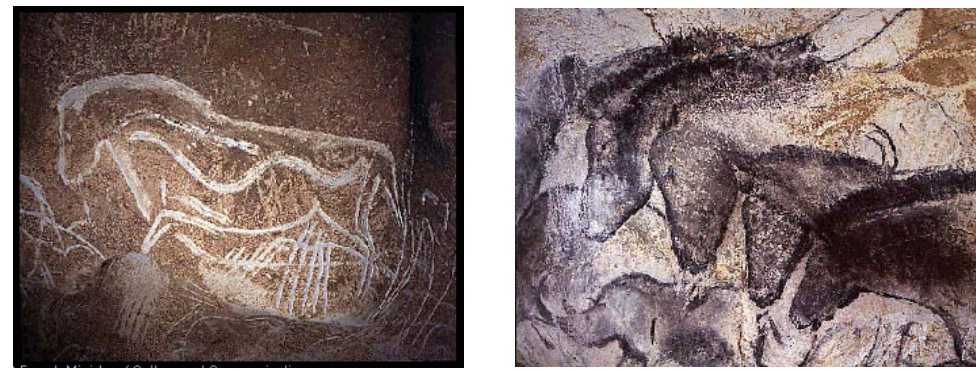

Figure 1: Prehistoric drawings (“Cave wall drawings," 2000)

This approach opened the way for what is called a character script, such as that of Chinese, Figure 2, in which each word is graphically represented by a separate symbol or by a sequence of such symbols ("Language," 2003).

$$
\begin{array}{ccc}
\text { 财 } & \text { 爱 } & \text { 吉 } \\
\text { Cai - money, wealth } & \mathrm{Ai}-\text { love, affection } & \mathrm{Ji} \text { - hope, (also: lucky) }
\end{array}
$$

Figure 2: Examples of Chinese ideograms

Chinese character writing has for many centuries been stylized, but it still bears marks of the pictorial origin. The ampersand (\&) sign, standing for "and" in English printing, is a good isolated example of a pictorial character used in an alphabetic writing system ("Language," 2003). Character writing is laborious to learn and imposes a burden on the memory. Alternative to it, is alphabetic writing.

The Greek alphabet came from the Phoenician script, a syllabic-type writing system that indicated the consonant sounds. The great innovation of Greek culture, the driving force behind the flowering of Greek philosophy, mathematics, science and culture was the addition of letters to represent the sounds of vowels (Ong, 1982). Before that, phonetically written languages provided letters only for consonant sounds. This led to a great deal of ambiguity in written text as readers had to interpret the letters and decide which vowel sounds to add, and where to add them. In Hebrew, this led to a culture of commentary on commentary on sacred texts. Authoritative Early Christian texts were written in Greek placing somewhat less demand on their interpretation. Another effect of Greek alphabetic writing was that the written language could immediately mirror changes in spoken language, and writers could coin new words that readers could pronounce unambiguously. Thus the new alphabetic Greek writing was a technology that 'standardised' words, removed ambiguity from writing; that is the quest expressed in this paper. The technology of the alphabet also made writing dynamic and contemporary an effect parallel to information technology develop- 
ments in our time. We must ask ourselves whether removal of ambiguity and dynamic sharing of information can lead to another great flowering of human culture.

Greek alphabet employs a set of letters standing for consonants and vowels. The Greek alphabet spread over the ancient world, undergoing numerous changes. From a Western version sprang the Latin (Roman) alphabet. Also derived from the Greek alphabet, the Cyrillic alphabet was devised in the 9th century ("Language," 2003).

Examples of Greek, Cyrillic and Latin alphanumeric symbols are shown in Figure 3 (note the name "Arabic" for ciphers used in English; in fact this system can be traced to Hindu mathematicians who also invented both the notion of and the symbol for value/amount 'zero').

Greek alphabet: $\Omega \vartheta \Psi \boldsymbol{\Sigma}$ Cyrillic alphabet: П Ц Б Д

\begin{tabular}{|c|c|c|c|c|c|c|c|c|c|c|c|c|}
\hline Latin cyphers & I & II & III & IV & V & VI & VII & VIII & IX & X & XI & L \\
\hline Arabic cyphers & 1 & 2 & 3 & 4 & 5 & 6 & 7 & 8 & 9 & 10 & 11 & 50 \\
\hline
\end{tabular}

Figure 3: Examples of Greek, Cyrillic and Latin alphanumeric symbols

Japanese employs a mixed system, broadly representing the roots of words by Chinese characters and the inflectional endings by syllable signs.

For the sake of indicating the diversity of means used for recording text (and numbers) two contemporary examples are presented in Figures 4 and 5.

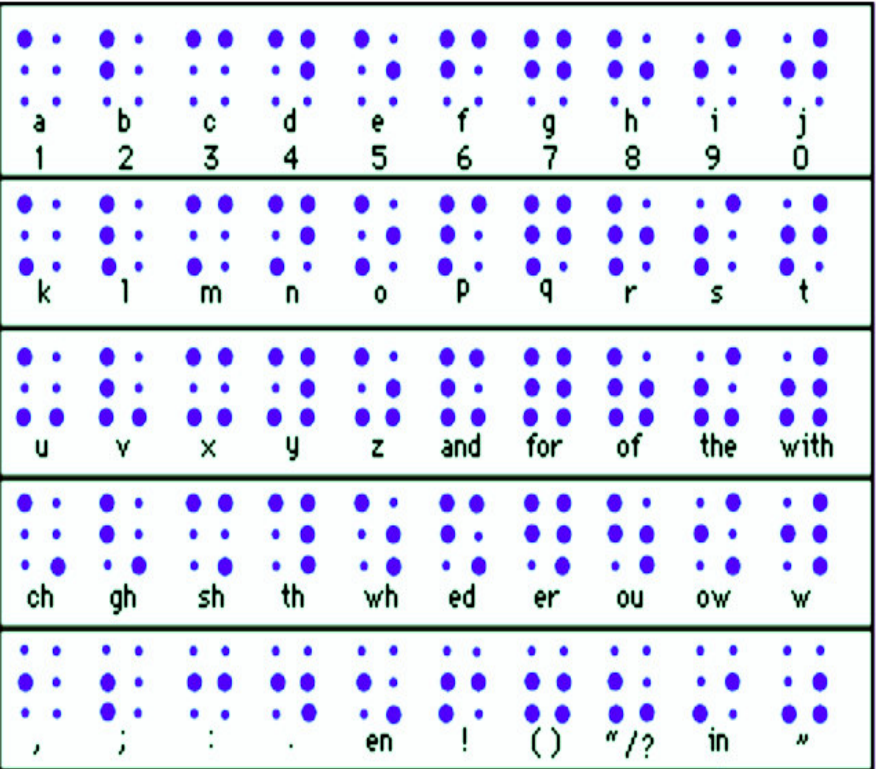

Figure 4: The Braille characters (embossed on paper and read by passing the fingers over the manuscript)

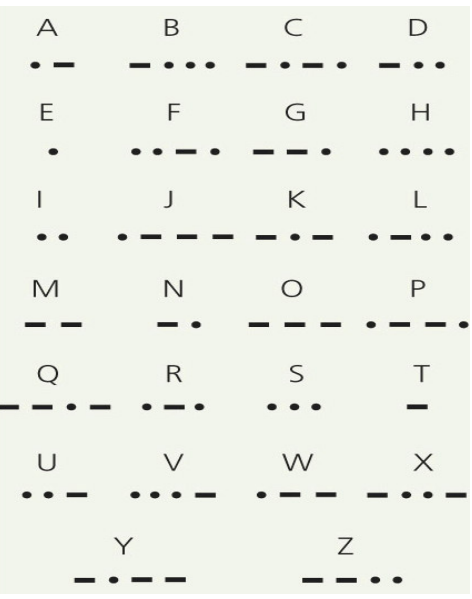

Figure 5: Morse Code for representing symbols by means of dots, dashes, and spaces, which can be transmitted as mechanical or electromagnetic pulses of varied lengths 


\section{Some Causes of Lexical Misinforming}

Somewhat special cause of misinforming may be attributed to use of homonyms, synonyms and acronyms.

On the other hand, it may be argued that, if all the synonyms and homonyms would be simply eliminated from the English language, the arts such as poetry and prose would be paralyzed and crippled. Efforts to resolve this conflict by creating completely artificial languages, present the evidence of the need for overcoming these dilemmas in 'naturally' developed linguistic structures. Amongst the numerous examples of homonyms and synonyms, one case of each category will be introduced to illustrate their impeding effect on knowledge transfer, at least to some extent. A convenient example is the term "figure" as defined in Merriam-Webster's Dictionary (2003), American Heritage Dictionary of the English (1992), Grolier Multimedia Encyclopedia (1997):

figure (n) : (i) a number symbol, (ii) numeral, (iii) digit, (iv) a geometric form (e.g. a line, triangle, or sphere) esp. when considered as a set of geometric elements (e.g. points) in space of a given number of dimensions, (v) a diagram or pictorial illustration of textual matter.

The first two above definitions, (i) and (ii), themselves can be taken as synonyms. The terms "figure" and "numeral" are synonyms, because both are defined in the same way as follows: "figure" ("numeral") is a conventional symbol (a figure or character) used to represent a number. The definitions under (iii), (iv) and (v) above, have different meanings, thus the terms "figure", attributed to each of these three cases appear to be homonyms.

Let us analyze another example - the term "turnaround"

A "general" meaning taken from The Free Dictionary (2003) has yielded the following descriptions:

i) turnaround - time need to prepare a vessel or ship for a return trip

ii) turnaround - a decision to reverse an earlier decision (synonyms: flip-flop, turnabout, reversal

iii) turnaround - an area sufficiently large for a vehicle to turn around

iv) turnaround - act or process of unloading and loading and servicing a vessel or aircraft for a return trip (synonyms: turnround)

v) turnaround - turning in the opposite direction (synonyms: turnabout, reversion, reversal, reverse)

Oxford dictionary (1989) provides the following definitions:

i) A space for vehicles to turn round in, often at the end of a drive

ii) The action or fact of turning round; a point in a team-game at which the teams change ends to play in the opposite directions.

Merriam-Webster's Online Dictionary (2003), provides for this term TURNAROUND (synonyms: TURNABOUT) the following definitions:

i) turnaround - the process of readying a vehicle for departure after its arrival especially without any intervening delays; also : the time spent in this process

ii) turnaround - the action of receiving, processing, and returning something

iii) turnaround - a space permitting the turning around of a vehicle 
Apart from this "general" homonymy, the further ambiguities begin when this term is applied in more specialized fields:

For example, a reputable source (Kelly, 1997) from the field of Maintenance Engineering ("a discipline concerned with ensuring and controlling the system reliability") states that, "The turnaround is an engineering event that takes place during a plant shutdown and involves the inspection, overhaul and, where necessary, the modification of existing equipment and the installation of new equipment."

It is certainly worth noting that another reputable source from the same field (Dunn, 2002) does not use term "turnaround" for this event, but prefers an expression "shutdown maintenance" (which requires twice as many symbols).

Merriam-Webster's Online Dictionary (2003) offers only one definition for term 'shutdown', namely "shutdown: the cessation or suspension of an operation or activity", which is virtually the same as in The Free Dictionary (2003) ("shutdown - termination of operations"), while the Oxford English Dictionary (1989) presents similar definition, just with more specific variations to a range of processes which all together fit in the above generic formulation.

Term "maintenance" itself is defined quite uniquely in all above mentioned sources: "maintenance - activity involved in maintaining (sustaining) something in good state (working order)".

Oxford English Dictionary (1989) converges to the same meaning: "Maintenance is the action of upholding or keeping in being a cause, right, state of things; (The action of keeping something in working order)."

A random search of Internet links the term "turnaround" to diverse domains of accounting, banking, law, management, psychology and information technology, basically within the scope of the above listed general meanings.

An obvious question is whether there is a rationale in using the shorter, but more ambiguous term "turnaround", or is it better to use twice as long term "shutdown maintenance" which may convey its meaning more clearly.

Yet another solution exists: we can introduce a completely new term with a unique meaning which is designed especially for that purpose. This solution raises questions about how the new term should be chosen and by whom. Should the discipline 'community of practice' manage the process? Do other languages have a better term? Will the adoption of such new terms lead to a decline in public understanding of science and technology in the light of the 'jargon' developing outside the dynamics of everyday language?

In addition to the above problems, the use of acronyms presents further hindrance to transfer of information. Acronyms are especially inconvenient for nowadays efforts to increase transparency and overcome the artificial boundaries between apparently disconnected disciplines.

Acronyms are brought in by the need for a frequent and rapid communication of combined and comprehensive information. In some cases, acronyms should be substituted by new, appropriately defined, terms. There, are of course, the cases where the use of acronyms is more appropriate than introducing a new term. For example - e.g. - is a good illustration of such a case.

However, let us observe another example. Decoding of acronym IT has yielded the following meanings:

$$
\begin{array}{ll}
\text { IT } & \text { - Industrial Technology } \\
\text { IT } & \text { - Information Technology } \\
\text { IT } & \text { - Intervalence Transition }
\end{array}
$$


The above three cases are quoted from Acronym \& Abbreviation Meanings (2003). The other reference ("The source for acronyms", 2003) provides the following meanings:

$$
\begin{array}{ll}
\text { IT } & \text { - Italy } \\
\text { IT } & \text { - Innovative tests } \\
\text { IT } & \text { - Information Transport } \\
\text { IT } & \text { - Interoperability Testing } \\
\text { IT } & \text { - Individual Therapy } \\
\text { IT } & \text { - Intensive Therapy } \\
\text { IT } & \text { - Inhalation Therapy }
\end{array}
$$

Since the last three acronyms are used in the same discipline (Medical Physiology) one may start to worry about the probability of being put on inhalation therapy while urgently needing an intensive therapy.

Could the multiplication in homonyms be avoided by ascribing a specific language to scientific disciplines, as was done by marrying Latin language to medicine? In this way, exaggerations in re-constructed terminology could be avoided (e.g., the term "methylpropenylenedihydroxycinnamenylacrylic acid" is obviously too lengthy). Furthermore, numerous concepts and phenomena are described by combinations of nouns and adjectives; this increases both the duration of information transfer and the physical space required for storage. The large terms could be conveniently replaced by shorter terms, purposely selected from the existing semantic base. Is there a substantial need for simultaneous usage of numerous languages in science and engineering? The translations obviously increase the number of formal records. An adoption of English in sciences has already taken place. However, an imperative is that the intrinsic trend of adopting foreign words into English language has to be continued. It would be an oversimplification to state that one language, with its unique vocabulary, can satisfy all diverse human interests (Spuzic \& O’Brien, 2002a).

The treasure of language variety is ready for building the bridges of knowledge across the abysses in this age of information technology.

\section{Some Cases of Semantic Misinformation}

Another significant aspect of ambiguity in knowledge transfer is the problem of definitions. This problem can be traced to the question of "defining the definition". Extensive studies are devoted to notions of definitions, e.g. within the disciplines of logic, informatics, philosophy and semantics, (Fetzer, Shatz \& Schlesinger, 1991; Kemerling, 2001a; Korpela, 2003; Robinson, 1963; Sager, 2000; Swartz, 1997), to mention just some reputable sources.

In spite of the fact that extensive bibliography is available, quite frequently, contemporary publications comprise ambiguous and homonymic definitions. This bad practice is especially damaging when it occurs in educational publications. For example Whitten and Bentley (1998) provide a number of "definitions" attributed to term "system analysis". According to this source, "System Analysis is...

- the dissection of a system into its component pieces to study how those component pieces interact and work

- the survey and planning of the system and project

- the study and analysis of the existing business and information system 
- the definition of business requirements and priorities for a new or improved system (a popular synonym is "logical design")

- the study of a business problem domain to recommend improvements and specify the business requirements for the solution."

The above formulations are ambiguous, for example:

i) The authors neglect the fact that the term "system" corresponds to a wider class of phenomena, more general than the limited group of information, business and production systems. Therefore, the application of the term "system analysis" to a limited domain, without respecting its more abstract nature, brings in vagueness and ambiguity in the subject.

ii) Terms "analysis" and "study" are complementary notions within an appropriate context, however term "planning" belongs to a different category of intellectual performance. Thus, it is not appropriate to use the formulation "...the planning of the system..." to define "the system analysis".

Although our realm is a stochastic phenomenon, our assortment of knowledge devices should comprise some deterministic tools. Namely, a language should be composed of unchanging terms - the definitions of these "permanent" terms cannot be changed dynamically, with the same rate that characterizes improvement of our knowledge. Indeed, there is a need for logical milestones, for definitions that serve as norms of the progress, as the reference points. Without a mathematically deterministic, static and rigid structure of language, it would be possible neither to establish the foundation of any knowledge nor to evaluate our hypotheses. New terms should be introduced in the language, rather than continuous modification of the established existing terms that are logically linked to fragments of our current knowledge. Homonyms and synonyms should be avoided, at least within one language - the scientific language.

At the same time, nothing stops us from preserving the language of arts. Natural evolution has equipped us with broad assortment of languages, thus enabling sufficient variety to preserve phenomena such as art, poetry and prose, the domains where language and terms escape beyond many restrictions.

Definitions of the concept of "definition" appear to be unsatisfactory. Let alone the general dictionaries, the specialized publications do not offer the acceptable formulations either. For example, Kemerling (2001b) presents the following formulation:

"Definition (is) an explanation of the meaning of a word. The five major kinds of definition (distinguished by the functions they may be used to perform) include:

$$
\begin{aligned}
& \text { - stipulative, } \\
& \text { - lexical, } \\
& \text { - precising, } \\
& \text { - theoretical, and } \\
& \text { - persuasive." }
\end{aligned}
$$

Explaining one term (definition) by simply introducing another term of the same category (explanation) is running in the loop (circularity). Classification on "five major kinds" (although it does help in studying this concept) does not complete the actual aim: to define the term "definition".

Swartz (1997), Kemerling (2001a) and Korpela (2003) provide detailed discussions on the meaning of this concept "definition". However they fail to acknowledge that, because of intrinsic infinity of both the universe and the matter forms, the complete, exhaustive (absolute) explanation of an arbitrary phenomenon is impossible. 
Such an approach is a consequence of unawareness of the actual dimensions of the universe, its eternity and infinity. In reality, every phenomenon may be searched infinitesimally deeper and infinitely broader providing that exhaustive explanations of all aspects are sought.

Some advances may be observed in the applied sciences. As known, we learn by doing things: many advances in physics come as a consequence of applications of solids in engineering while mathematical statistics developed rapidly to provide predictive control in process engineering. As a further example, disciplines such as Information Technology and Computer Sciences have demanded more formal definitions of terms such as "Information".

According to International Committee for Information Technology Standards (2003), "information is (1) The meaning that is currently assigned to data by means of the conventions applied to these data. (2) In information processing, any fact, concept, or meaning derived from data and associated context or selected from knowledge. (3) In a conceptual schema language, any kind of knowledge about things, facts, or concepts of a universe of discourse that is exchangeable among users. (4) In information theory, knowledge that reduces or removes uncertainty about the occurrence of a specific event from a given set of possible events.

- Universe of discourse is: in a particular context, all entities that are of interest. A universe of discourse may include many entity worlds, possibly including entities that are not yet perceived or considered; for example: if the area of interest is 'finance', the universe of discourse could be 'all financial aspects of an organization'.

- Knowledge is: (1) An organized, integrated collection of facts and generalizations. (2) Information representing human experience and expertise."

The development of artificial intelligence for storing the records and for information search, has stimulated numerous questions related to terminology and semantics. The principles developed in computer sciences can also be applied to other methods of expressing the structure of information, e.g. for defining the structure of records in a data base of definitions. In hypertext, a good definition would contain hyperlinks for cross-references to other definitions and perhaps links to additional information such as encyclopedia articles and illustrative examples (Korpela, 2003).

It might be useful to add a topic attribute that indicates the realm where the definition applies, such as general, biology, history etc. Probably all the attributes should be optional, in a general format of definitions. This would make it possible to search for definitions for a common word when it is used as a term in some special discipline. However, it would be very difficult to define a standardized and widely useful categorization, so this idea needs to be elaborated. It might be possible to define a generic method of specifying a topic hierarchically and to start by defining a very coarse standardized top-level categorization (e.g. based on a study of categories used in dictionaries and grouping them to more general terms). These dictionary entries would contain usage examples, illustrations, and other material as part of a definition (Korpela, 2003).

The above formulations of the concept of definition require to be further improved. Therefore an attempt is made herewith to devise a better definition of the term "DEFINITION".

\section{A Contribution to Defining the Term 'Definition'}

Language and terminology can be structured by following the strict mathematical rules. For example, Mathematical Linguistics is dedicated to the study of the formal properties of language. Numerous universities consolidate the formal study of language under the transdisciplinary umbrella of cognitive sciences, supported by the auxiliary fields of mathematics and artificial intelligence. 
Each definition should be attributed a unique set of terms and symbols. Language is alive, however it lives through everlasting growth by introducing new definitions. Some old definitions may prove to be fallacies in future, but the awareness of these misconceptions also constitutes knowledge and deserves to be permanently recorded.

Bearing in mind the diversity of symbols and variety of languages it appears that there are no limits to introducing as many new terms and definitions as our growing knowledge will require.

In addition to attributing a unique set of words to a concept (term) to be defined, a definition must satisfy the following requirements:

i) The infinity of universe and the endless variety of matter forms have been postulated by number of authors (Spuzic \& O'Brien, 2002). This realm must be acknowledged by incorporating a scope (artificial boundaries) into formulation of definition. The axioms and assumptions must not be omitted.

ii) Our understanding of something is fundamentally affected by the motives that have prompted us to understand some phenomenon. This also must be acknowledged within a definition. One way to do this is to specify the "minimum purpose statement".

iii) A definition is not limited to use of alphanumeric symbols only, it may be combined with figures, sound and other means.

\section{Minimum Purpose Statement}

The following definition of a term "DEFINITION" is made to at least enable a decision to be made about whether an item of information, interpretation, statement, conclusion, explanation, concept, cognition, theory, etc., qualifies to be identified as definition which contributes to formation and application of the knowledge.

Assumptions and axioms:

- $\quad$ Except for the term "DEFINITION", all other terms (words) that are used within this text, where the definition of the term "DEFINITION" is presented, are commonly known and understood. Still, for the sake of clarity, the following explanations are added:

- $\quad$ "SOMETHING" is a phenomenon of a most general meaning.

- $\quad$ An "ENTITY" is an element that belongs a broader category: the "PHENOMENA".

- $\quad$ A "RELATION" is something that holds between two or more phenomena. "RELATION" is an aspect (something) that connects (relates) two or more phenomena.

\section{Definition}

Short version: A DEFINITION is a form of some relation that significantly increases the probability of occurrence of some premeditated change of some relation.

More detailed version: A "DEFINITION" is a form (an appearance of something as distinguished from the substance of which it is made; a pattern, a record, a representation, a model, an imprint, a reproduction), of some relation(s) that significantly increases probability of realization (occurrence) of a pre-selected (premeditated) change of some relation to be achieved by an entity that is capable of utilizing this definition for such a specified purpose. A definition cannot be generated (invented) without an entity, a system (material form), which exists at certain level of higher order and implies that the chaos within its domain is suppressed to a certain minimum degree. An example of such an entity is a human being. Other examples are some advanced levels of artifi- 
cial intelligence systems. However, once it is generated, a definition can continue to exist (to be recorded) without the existence of the mentioned entity.

A definition should be complemented with a minimum purpose statement (i.e., an explanation about a minimum domain of purposes for which the presented definition can be used). This statement does not exclude the possibility of using the definition correctly for some other purposes, it only specifies at least some minimum domain where the definition is applicable. However, this extended use must not violate (contradict to) already attributed meaning. In addition, this extended use must not cause synonymy or homonymy.

It is useful if a definition is accompanied by the set of axioms that delimit the initial assumptions. If two definitions are mutually contravening, one of them should be eliminated from the class of definitions. Such disqualified information should be included in the class of assumptions or hypotheses, or, if probability of erroneousness is high, it should be classified as a misinformation (misconception, fallacy).

For example, erroneous information or a mistaken theory do not qualify to be identified as definitions. For undecided information, terms "measurement", "notion", "signal", etc. can be used with a statement indicating whether they are proven to be true, including the confidence indicator. A hypothesis should not qualify to be a theory until it can be proven with specified probability. For example, in information technology, the bytes are recording elements that become information only when they provide definition.

The scientific language should attribute to each definition a unique set of words (Spuzic \& O’Brien, 2002b).

Let us return to the ambiguous term "figure" referred to earlier. We shall define it now in a different way. (The minimum purpose statement:) The following definitions are presented to provide several examples how ambiguity due to appearance of synonyms and homonyms can be avoided.

figure (n): an arrangement of points made within two-dimensional space to present an impression, a visual static model of something (e.g., a figure printed on a book page, showing a front view of a home)

digit (n): a figure representing a numeral

numeral (n): any of the elements that can be combined to form numbers in a number system (e.g., decimal system, binary system, hexadecimal system, etc.). Examples: "0", "1", "A" ("A" is listed under an assumption that there is a convention within a non-decimal system, attributing a status of certain numeral to "A"). Different combinations of numerals provide different numbers.

For the sake of explicitness, the term denoting "thing itself" is here distinguished from the "model representing something": thus "digit" is a "model representing a numeral"; the "numeral" is the "thing itself". Furthermore, "numeral" is only an element of a more complex structure - a "number":

number (n): an element belonging to a mathematical system, so-called number system. For example, "1", "200", "0.0003", "1001100001", "I", "p", "0", etc. In other words, number is a mathematical measure - a norm. More generally, number is an element of mathematics. More specifically, number is a mathematical element that can be subject to mathematical operations (e.g., succession, addition, and multiplication) following mathematical logic. More detailed knowledge of numbers is comprehended within various branches of mathematics and logic (Spuzic \& O'Brien, 2002b).

To conclude this excursion into the world of words, we shall introduce a further complementary term defining the "model representing the number", namely a "cypher": 
cypher (n): a figure presenting a number; a combination of digits used to depict a number. In addition, most authors ignore the fact that definition can be complemented by figures or by other means.

Consider the case of term "square". Here are five competing candidates, i.e. sets of logically necessary and jointly sufficient conditions introduced by Swartz (1997):

"square" = "a plane closed figure that has exactly four sides all of which are straight and equal to one another and whose interior angles each measure $90^{\circ}$

"square" = "a plane closed figure having four straight sides and whose diagonals are both equal in length to one another and bisect one another at right angles"

"square" = "a straight-sided, plane, closed figure, every diagonal of which cuts the figure into two right isosceles triangles"

"square" = "an equilateral parallelogram containing no (interior) acute angles"

"square" = "an equilateral parallelogram containing four axes of symmetry".

The above definitions will be greatly elucidated by adding a simple figure denoted "square", Figure 6.

Mathematics is a good model of a structure where ambiguities are suppressed and there is no room for dual meaning of terms. The analogies between English language grammar and mathematics were frequently observed. "Naturally" developed structures and interactions that appear within the language are certainly valuable phenomena that deserve detailed study not only within the linguistics but also within other disciplines. Nonetheless, improvement is possible, and indeed, rather than waiting for natural events to bring the troublesome problems to the surface, it is rational to anticipate and avoid the potential hindrances. An analogy with mathematical structures suggests a useful strategy in resolving the problems of synonyms and homonyms. For example, the problem known as "the word problem for groups" states that a given presentation of a group is said to have a soluble word problem if there exists a method enabling one to decide for each pair of words in a finite number of steps whether or not they represent the same element. The word problem has been solved for some simple classes of groups, but it has been shown to be insoluble in general group representations. Presentations can be obtained for algebras in any variety, and for many algebras encountered in practice, a presentation can be found with soluble word problem. Therefore, if we apply the rules of algebra to one explicit language (e.g., English), it is possible to define it in such a way that only the presentations with soluble word problem do appear. In short terms, it should be established a formal structure that attributes to each definition a unique set of words.

However, at the same time one cannot ignore the fact that another, more general, algebra (language) will continue to exist, where the insoluble word problems will remain (Spuzic \& O'Brien, 2002b).

\section{Presentation}

Knowledge is based on perceptions and thoughts, and the translation of perceptions and thoughts in the language is perhaps one of the most challenging aspects of the transfer of knowledge. Development and structure of languages are most intriguing phenomena, and inasmuch as there is an obvious link between language and thinking, much remains to be understood about this correlation. The natural, spontaneous development of languages should not be interrupted - there is much to learn from this evolution. Yet the modifications and improvements within the controlled 
language structure - the scientific language - should be considered. A false notion might initially appear, that by introducing newly constructed terms, we only emphasize the problem of the voluminousness of the knowledge. On the contrary, in the context of the actual increase in knowledge, introduction of efficacious new terms can help to promote more rapid communication of knowledge.

From the distant 19th Century, the words of Rudolph Clausius come to us as an inspiration, an example how this kind of problems can be dealt with: "I propose... to cal S the entropy of a body, after the Greek word 'transformation'. I have designedly coined the word 'entropy' to be similar to energy, for these two quantities are analogous... that an analogy of denomination seems to be helpful". Helpful for what, if not - helpful for the transfer of knowledge (Serway, 1996).

Assuming that all terms needed for information transfer are defined appropriately, there are still aspects that remain to be improved in order to optimize education, knowledge records and even ongoing communication.

It is clear that the combination of figures and text provides efficient means for recording knowledge. In fact, this combination is often more perceptible than direct observation of the phenomenon itself.

Apparent limitations of any specific writing system should not be confused with limitations of transporting the thoughts by means of words and script altogether. Namely, the concept of ideograms can be exploited far beyond the limitations of Chinese, Japanese, or other known ideographs. An ideograph, or ideogram, is a character or figure symbolizing the idea of a thing, without expressing the name of it. Ideograph is a special case of figure - basically it is a figure or symbol that carries on an idea.

Mathematicians use the broad variety of symbols, and they do not hesitate to invent new symbols when they need them, e.g.

$$
\Sigma, \quad \Pi \quad, \quad \text { and } \supseteq
$$

A B

$$
\mathrm{ydx} \mathrm{dz}
$$

$0 \quad 0$

Vigorous advance of writing systems and certain semantic tools, such as metonymy, come to being due to efforts to overcome technical limitations of printing media and modes. However the recent computer revolution has removed many of these barriers. Communication of knowledge is today eased by using the contemporary means for recording and presenting information (multimedia).

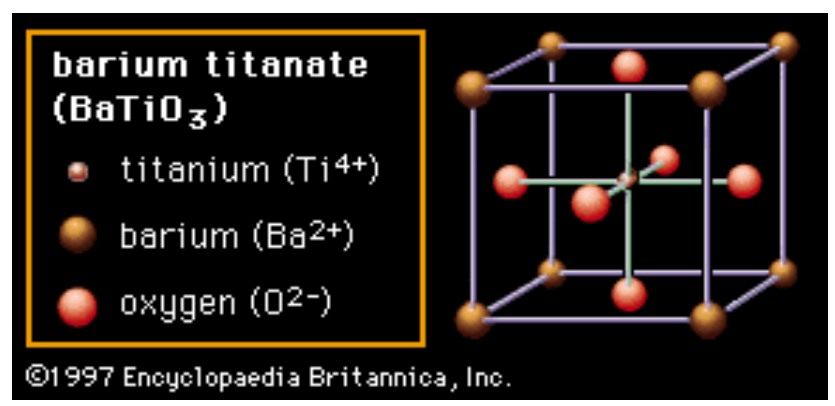

Figure 7: The arrangement of titanium, barium, and oxygen ions in barium titanate $\left(\mathrm{BaTiO}_{3}\right)$; an example of the perovskite crystal structure ("Ceramic composition”, in Encyclopedia Britannica Online, 2003) 
The application of graphs and figures has enormous impact in knowledge transfer in education. Presenting to students a selection of figures in the course of lectures is very stimulating. Mathematical models and equations, however powerful in modeling the laws and relations, should be abundantly complemented by illustrations. Any engineer will agree that no text can substitute for appropriate sketches, diagrams, layouts and other figures. The meaning of the attribute "appropriate" includes not only the quality of being correct, but equally importantly, the figure should be clear, instructive, and inspiring (Spuzic \& O'Brien, 2002). Figures 7 to 9 illustrate these ideas.

Notions of geometry have brought in enormous advances in sciences. For example in mathematics, fractal geometry, with its concepts of self-similarity and non-integer dimensionality, has been applied increasingly in statistical mechanics, notably when dealing with physical systems consisting of seemingly random features. For example, fractal simulations have been used to plot the distribution of galaxy clusters throughout the universe and to study problems related to fluid turbulence. Fractal geometry also has contributed much to computer graphics. Fractal algorithms have made it possible to generate lifelike images of highly complicated matter forms, Figure 9.

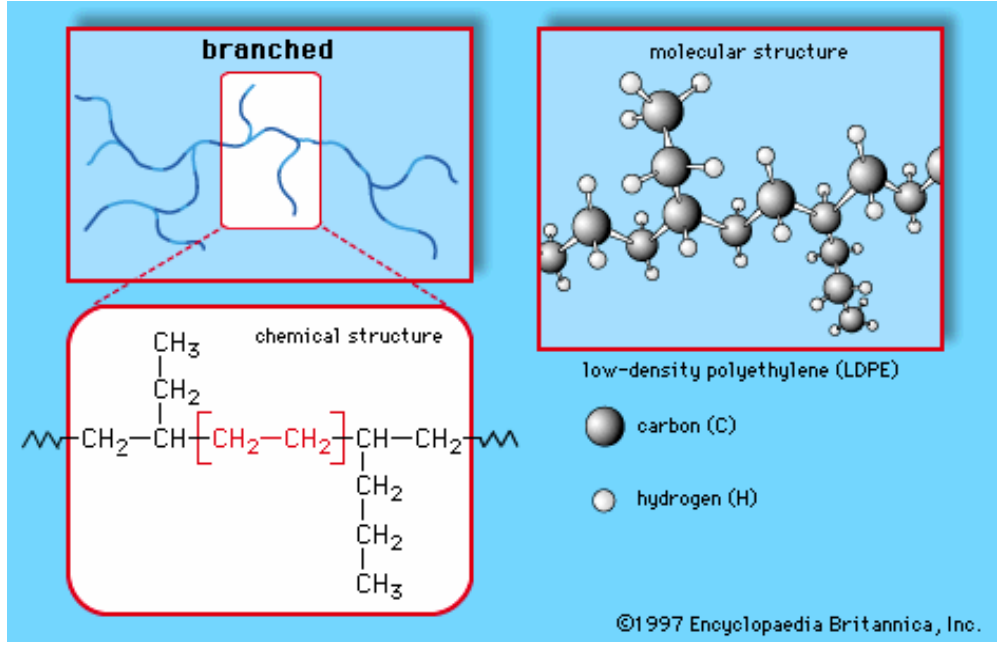

Figure 8: A high-density polyethylene-linear polymer structure ("Industrial polymers", in Encyclopedia Britannica Online, 2003)
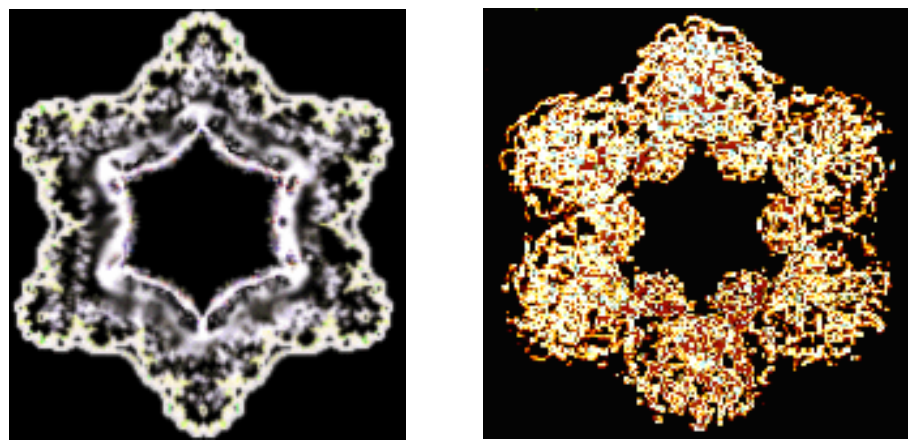

Figure 9: The structure of ornithine decarboxylase as resolved by (left) the computer manipulated Mandelbrot Seventh fractal and (right) x-ray crystallography (adjusted from Sharp \& Brown, 1995) 
Knowledge can be presented in a structured manner - within a framework of layers and levels - following an analogy to the construction of matter itself. These 'knowledge layers' should be connected via key terms: a good example of presenting the knowledge in such a structured manner is the Encyclopedia Britannica Online where the terms are linked by means of the Hyper Text Markup Language. There are no limits to linking and rearranging the selected fragments of knowledge in order to follow the specific topic (Korpela, 2003; Spuzic, 1999).

\section{Conclusion}

The quantity of the knowledge increases with geometric progression and the corresponding body of records grows even faster. Transferring knowledge and pertinent information has become a vigorous activity. An increasing need for de-specializing the knowledge, due to an increase in inter-disciplinary communication, calls for more friendly i.e. more detailed, hence even more voluminous, communication. Definitions are fundamental bricks of knowledge; however it appears that the notion of definition itself is not sufficiently explained.

There is no doubting the enormous advantages of the existence of variety of languages - the question is how this treasure can be made more useful.

Vague definitions, homonyms and synonyms contribute significantly to misinformation, and are especially harmful in activities such as education. Therefore in this work, the relevant aspects of misinforming are analysed and recommendations are made on how defining process can be improved.

The infinity of micro and macro-cosmos must be taken in account by incorporating a scope (artificial boundaries) into formulation of definition. The axioms and assumptions must not be omitted from a definition.

Our understanding of something is fundamentally affected by the motives that have prompted us to explore it. This also must be acknowledged within a definition. One way to do this is by specifying the "minimum purpose statement" along with each definition.

Even when homonyms and synonyms are eliminated from the scientific language, it is useful to add to a definition a topic attribute that indicates the realm where the definition applies, such as "general", "zoology", "ancient history" etc.

A properly formulated definition is a probability intensifier. However, in the case of ambiguous definition, the desired effect becomes more uncertain and the probability of misinforming increases.

Definitions are not limited to lexical formulation, and they are indeed more clear and useful when combined with other means of transferring ideas, e.g. figures. Nowadays artificial intelligence aids (facilities such as computers) have overcome the limitations present in the "classical" printing media. Therefore, this paper proposes that knowledge is best presented by combining textual definitions with appropriate multimedia formats e.g. with figures, sound, calculations, animations, and other means of processing information.

\section{References}

Acronym \& abbreviation meanings - Business \& technical. (2003). Retrieved December 8, 2003 from http://acronym.langenberg.com/

American heritage dictionary of the English language (3rd ed.). (1992). Houghton Mifflin Company. Electronic Version 1995, Softkey International Inc.

Bolter, J. D. (2001). Writing space: Hypertext and the remediation of print. Lawrence Erlbaum Assoc. 
Cartelli, A. (2003). Misinforming, misunderstanding, misconceptions: What informing science can do. Pro ceedings of Informing Science + IT Education Conference, Pori, Finland June 24-27, 2003, 12591273. Retrieved March 7, 2004 from http://ecommerce.lebow.drexel.edu/eli/2003Proceedings/docs/156Carte.pdf

Cave wall drawings from the Chauvet Cave. (2000). French Ministry of Culture and Communication; Regional Direction for Cultural Affairs - Rhône-Alpes region; Regional department of archaeology. Retrieved August 1, 2000 from http://www.culture.fr/culture/arcnat/chauvet/en/album.htm

Ceramic composition and properties. In Encyclopadia Britannica Online. Retrieved December, 3, 2003, from http://www.britannica.com/eb/article?eu=114695

Driscoll, M. P. (2000). Psychology of learning for instruction. Boston: Allyn \& Bacon.

Dunn, S. (2002). Maintenance terminology - some key terms. Plant Maintenance Resource Center. Retrieved December, 3, 2003, from http://www.plant-maintenance.com/terminology.shtml

Fetzer, J. H., Shatz, D. \& Schlesinger, G. N. (Eds.). (1991). Definitions and definability: Philosophical perspectives. (Synthese Library, 216), n.a: Kluwer Academic Publishers.

Grolier Multimedia Encyclopaedia. Version 9.01M. (1997). Grolier Interactive Inc.

Habermas, J. (1996). Knowledge and human interests: A general perspective. In W. Outhwaite (Ed.), The Habermas Reader. Cambridge: Polity Press.

Industrial polymers, chemistry of. In Encyclopadia Britannica Online. Retrieved December, 3, 2003 from http://www.britannica.com/eb/article?eu=115415

InterNational Committee for Information Technology Standards. Retrieved December, 5, 2003 from http://www.ncits.org/ and http://www.ncits.org/tc_home/k5htm/i2.htm\#information

Jakupec, V. (1996). The Nature of Education and Perspectives on Teaching and Learning. In Teaching and Learning in Open and Distance Education 2. Study Guide, Adelaide: University of South Australia and Deakin University.

Kahneman, D. \& Frederick, S. (2001). Representativeness revisited: Attribute substitution in intuitive judgement. Preprint of chapter to appear in T. Gilovich, D. Griffin \& D. Kahneman (Eds.), Heuristics of intuitive judgement: Extensions and applications. New York: Cambridge University Press.

Kelly, A. (1997). Maintenance organization and systems. Oxford: Butterworth-Heinemann.

Kemerling, G. (2001a). Definition and meaning, Retrieved December 3rd 2003 from http://www.philosophypages.com/lg/e05.htm\#kinds

Kemerling, G. (2001b). A dictionary of philosophical terms and names. Retrieved December, 4, 2003 from http://www.philosophypages.com/dy/

Korpela, J. (2003). Definition: A definition and an analysis, Retrieved December, 3, 2003, from http://www.cs.tut.fi/ jkorpela/def.html

Language. Encyclopadia Britannica from Encyclopædia Britannica Premium Service. Retrieved December, 2, 2003 from http://www.britannica.com/eb/article?eu=114866

Lovat, T. \& Smith, D.L. (1995). Curriculum: Action on reflection revisited ( $3^{\text {rd }}$ ed.). Sydney: Social Science Press.

Merriam-Webster's Online Dictionary. (2003). In Encyclopadia Britannica Online. Retrieved December 1, 2003, from http://www.britannica.com

Ong, W. J. (1982). Orality and literacy: The technologizing of the word. London: Methuen.

Robinson, R. (1963). Definition. Oxford: Oxford University Press.

Sager, J. C. (Ed.). (2000). Essays on definition (Terminology and lexicography research and practice), Amsterdam: John Benjamins Publishing Co. 
Serway, R. A. (1996). Physics for scientists \& engineers with modern physics. Philadelphia: Saunders Col lege Publishing.

Sharp J. A. \& Brown, Jr., R. M. (1995). Atomic and molecular imaging with the transmission electron microscope. ASPP poster (Charlotte, N.C., August, 1995); Department of Botany, The University of Texas at Austin. Retrieved March 17, 2001 from http://www.botany.utexas.edu/facstaff/facpages/mbrown/ongres/jsharp.htm

Spuzic, S. (1999). An initiative in improving knowledge transfer in engineering education. Proceedings from the 2nd Asia-Pacific Forum on Engineering and Technology Education, Ed. Z. Pudlowski, The University of Sydney, 4-7 July 1999, UICEE, p. 41.

Spuzic, S. \& O'Brien, J. (2002a). Mobilizing the curiosity, attention, and inventiveness of future materials engineers, Part I: The infinite variability of matter, Journal JOM-e, July 2002, a publication of The Minerals, Metals \& Materials Society (TMS). Retrieved December, 1, 2003, from http://www.tms.org/pubs/journals/JOM/0207/SpuzicI/SpuzicI-0207.html

Spuzic, S. \& O'Brien, J. (2002b). Mobilizing the curiosity, attention, and inventiveness of future materials engineers, Part II: A fascinating vision. Journal JOM-e, July 2002, a publication of The Minerals, Metals \& Materials Society - TMS. Retrieved December, 1, 2003, from http://www.tms.org/pubs/journals/JOM/0207/SpuzicII/SpuzicII-0207.html

Swartz, N. (1997). Definitions, dictionaries and meanings. Department of Philosophy, Simon Fraser University. Retrieved, December 3, 2003, from http://www.sfu.ca/philosophy/swartz/definitn.htm

The source for acronyms and abbreviation. Stands.com. Retrieved December, 7, 2003, from http://www.stands4.com/browsesearch.asp

Whitten, J. L. \& Bentley, L. D. (1998). Systems analysis and design methods (4th ed.). Boston: Irwin/ McGraw-Hill.

\section{Biography}

Dr Sead Spuzic is a Senior Lecturer at the Central Queensland University. He has pursued his career within the interface of Industry and Academe with particular focus on manufacturing systems. He has studied and worked in Australia, Middle East and Europe. His professional interests are best presented by means of his publications, which are listed on the internet website http://www.geocities.com/belaliki/welcome.html. Dr Spuzic has focused recently on producing multimedia educational materials - he believes that the future of education is inseparable from applications of artificial intelligence and information science.

Fons Nouwens is a lecturer in the Learning Evaluation Innovation and Development (LEID) center at Central Queensland University at Rockhampton campus. He has degrees in engineering and education and has been a teacher in engineering and an instructional designer for 30 years. He is currently studying for a Master of Educational Technology with the University of British Columbia and has scholarly interests in educational transitions (school to university, university to work, work to university), development of graduate attributes and identity, lifelong learning and distributed cognition. 